\title{
ANALYSIS OF THE DENSITY OF ELECTRON-HOLE PAIRS FOR MINIMAL PUMP ENERGY IN A LASER DIODE WITH COHERENT FEEDBACK
}

\author{
M. A. GRADO-CAFFARO* and M. GRADO-CAFFARO \\ Scientific Consultants, C./Julio Palacios, 11, 9- B, 28029-Madrid, Spain
}

(Received 22 June 1998; In final form 4 September 1998)

The average value in the time domain of the density of electron-hole pairs in a semiconductor laser with coherent feedback is calculated under conditions of minimal pump energy. This value is found to be finite; the significance of this result is discussed as well as the asymptotic value of the above carrier density.

Keywords: Density of electron-hole pairs; laser diode; coherent feedback; minimal pump energy

\section{INTRODUCTION}

Optical coherent feedback is a relevant phenomenon in the context of semiconductor lasers; this phenomenon is strongly connected with AM and FM noises and coherence collapse, and a number of questions related to this subject remain open from the theoretical point of view since a considerable part of the literature only contains empirical techniques which, in practice, have achieved a scant success. However, in recent years, some authors as Refs. [1-4] have obtained important results; in particular, we refer to AM and FM noises and minimal pump energy. This last subject has been treated in Ref. [4] in a manner that can be considered as a singular source of fruitful future investigations within the frame of optical coherent feedback in laser

${ }^{*}$ Corresponding author. 
diodes. At this point, we can claim that the aim of the present paper is to analyze the electron-hole density in a semiconductor laser with coherent feedback under conditions of minimum pump energy; these conditions have been considered in Ref. [4]. The relevance of pump energy minimization is clear since minimum pump energy corresponds to maximum quantum efficiency and it is obvious that this optimal efficiency represents a crucial problem.

\section{THEORETICAL CONSIDERATIONS}

For minimal pump energy in a laser diode with coherent feedback, the density of the electron-hole pairs $n(t)$ is given by the following relation (see Ref. [4]):

$n(t) \approx\left(\frac{n_{0}-\Gamma_{0} P_{s} T}{1+T \zeta P_{s}}\right) \exp \left[-\frac{1}{2}(\sqrt{5}+1)\left(T^{-1}+\zeta P_{s}\right) t\right]+\frac{\left(\Gamma_{0}-\zeta n_{0}\right) P_{s}}{T^{-1}+\zeta P_{s}}$

where $t$ stands for time, $n_{0}=n(0), T$ is the lifetime for spontaneous recombination of electrons and holes in the active layer, $\Gamma_{0}$ is the rate of optical power emission, $\zeta$ is the gain coefficient, and $P_{s}=|E|^{2}$ is the density of photons in the active layer; $E(t)$ is the slowly varying envelope of the complex electric field in the active layer. Here, harmonic solutions to Eq. (2) of the form $E(t)=\sqrt{P_{s}} \mathrm{e}^{i \Omega t}$ are considered so that $P_{s}$ does not depend on time (see previous refs.); $\Omega$ denotes feedback-induced frequency shift. The above mentioned Eq. (2) is the following Lang-Kobayashi equation namely [5]:

$$
\frac{d}{d t}(E(t))=\frac{1}{2}\left[G(n(t))-\Gamma_{0}\right] E(t)+\gamma E(t-\tau) \exp \left(-i \omega_{0} \tau\right) \quad(i=\sqrt{-1})
$$

where $\omega_{0}$ is the optical angular frequency which is near the laser angular frequency, $\gamma$ is the feedback rate, $\tau$ is the feedback delay time, and $G(n(t))$ is the complex gain function which depends upon $n(t)$. Now, from Eq. (1) we get:

$$
n_{\infty} \equiv \lim _{t \rightarrow \infty} n(t)=\frac{\left(\Gamma_{0}-\zeta n_{0}\right) P_{s} T}{1+\zeta P_{s} T}
$$


On the other hand, note that also from Eq. (1) it is deduced the existence of a relaxation time given by:

$$
\tau_{r} \approx \frac{2 T}{(1+\sqrt{5})\left(1+T \zeta P_{s}\right)}
$$

so that from Eqs. (3) and (4) it follows:

$$
n_{\infty} \approx 1.62 P_{s} \tau_{r}\left(\Gamma_{0}-\zeta n_{0}\right)
$$

Next we shall evaluate the average value of $n(t)$ as follows:

$$
\langle n\rangle=\lim _{t_{0} \rightarrow \infty}\left[\frac{1}{t_{0}} \int_{0}^{t_{0}} n(t) d t\right]
$$

Then, by taking into account formulae (1) and (6) as well as L'Hôpital's rule with respect to parametric differentiation, we obtain:

$$
\begin{aligned}
\langle n\rangle= & n_{\infty}+\left(\frac{n_{0}-\Gamma_{0} P_{s} T}{1+T \zeta P_{s}}\right) \\
& \times \lim _{t_{0} \rightarrow \infty} \exp \left[-\frac{1}{2}(1+\sqrt{5}) \times\left(T^{-1}+\zeta P_{s}\right) t_{0}\right]=n_{\infty}
\end{aligned}
$$

Notice that result (7) coincides with $n_{\infty}$ (see formula (3)), that is, timeaveraged electron-hole density is exactly equal to the asymptotic value of this density in the time domain.

\section{DISCUSSION}

We have found that $\langle n\rangle=n_{\infty}$, this value being dependent on relaxation time $\tau_{r}$; at this point, note that $\tau_{r}$ does not depend on the feedback delay (for details on this delay, see Refs. [2, 4]). In addition, we must emphasize that our results refer to minimal pump energy which constitutes a very interesting circumstance since this situation is related to crucial problems in the context of chaotic phenomena. It is well-known that these phenomena are not at present well understood so very important research efforts are needed. Finally, we can mention Refs. $[5,6]$ as basic work in this context. 


\section{References}

[1] Grado-Caffaro, M. A. and Grado-Caffaro, M. (1994). Act. Pass. Electronic Comp., 16, $101-103$.

[2] Grado-Caffaro, M. A. and Grado-Caffaro, M. (1994). Optik, 98, 74-78.

[3] Grado-Caffaro, M. A. and Grado-Caffaro, M. (unpublished).

[4] Grado-Caffaro, M. A. and Grado-Caffaro, M. (1994). Mod. Phys. Lett. B, 8, $819-822$

[5] Lang, R. and Kobayashi, K. (1980). IEEE J. Quantum Electron, QE, 16, 347.

[6] Yasaka, H., Yoshikuni, Y. and Kawaguchi, H. (1991). IEEE J. Quantum Electron, $Q E, 27,193-204$. 

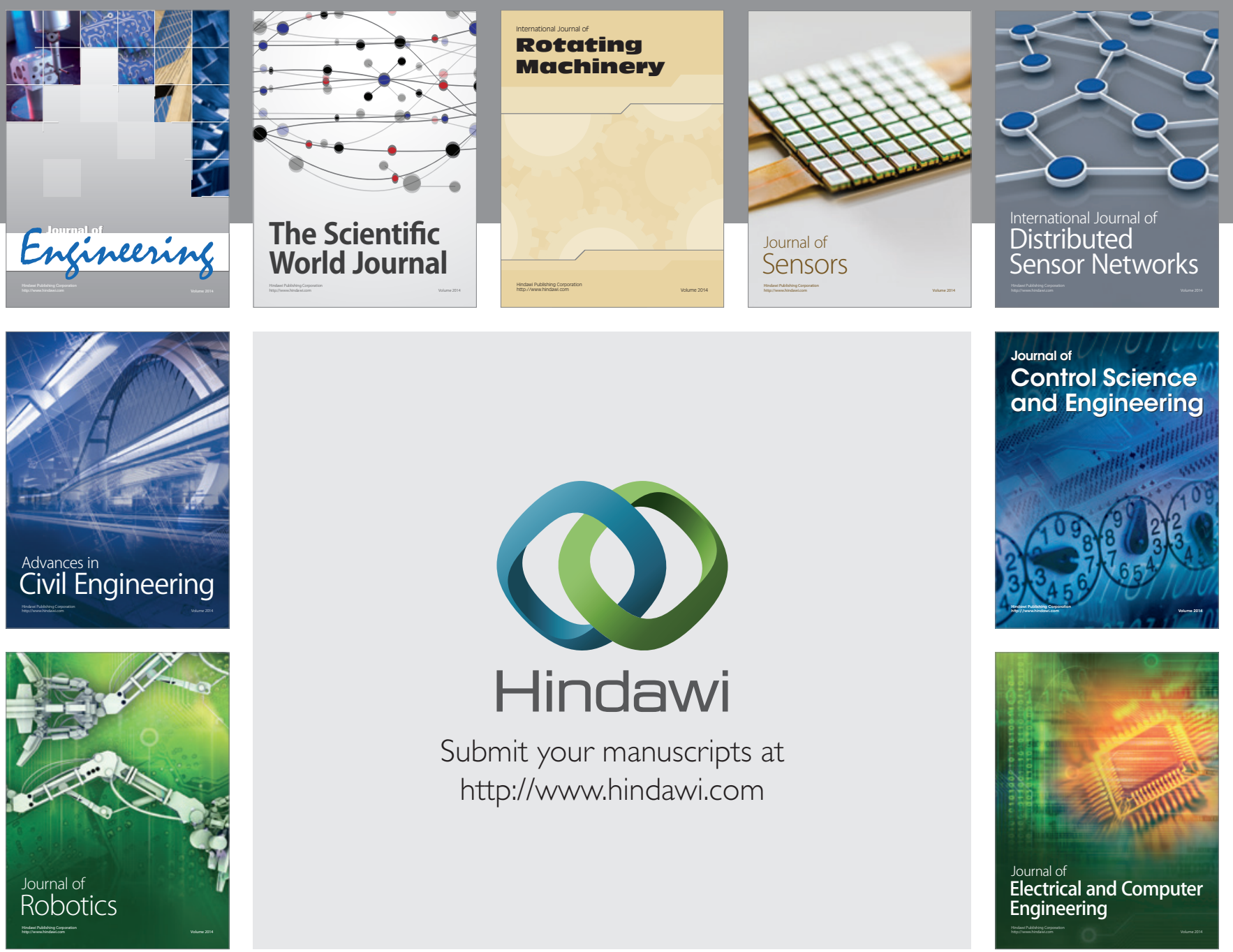

Submit your manuscripts at

http://www.hindawi.com
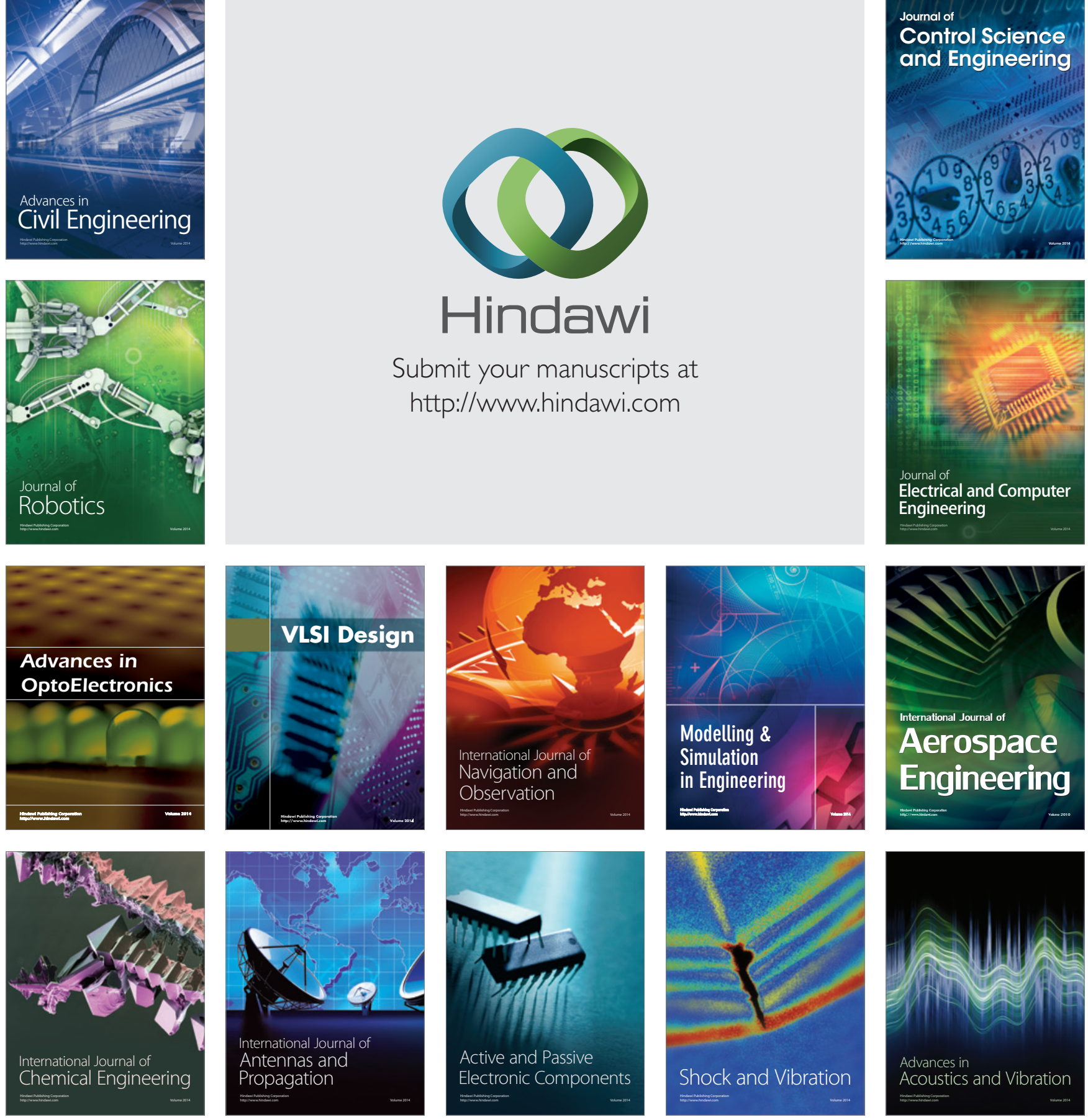